\title{
Más allá de la disciplinariedad. Elementos para pensar la Pedagogía como construcción transdisciplinar ${ }^{2}$
}

\author{
Beyond the Discipline. \\ Elements to Think the Pedagogy as a Transdisciplinary Construction
}

\begin{abstract}
Resumen
La crisis de los modelos hegemónicos de conocimiento y las demandas de la compleja realidad educativa ponen en entredicho el trabajo de las ciencias de la educación, en tanto que se enmarcan en el paradigma de la simplicidad en su expresión disciplinar. En este artículo se reflexiona acerca de los modos en que se ha abordado la comprensión de la educación y se presentan elementos para pensar una Pedagogía desde la complejidad, a saber, una Pedagogía como construcción transdisciplinar.
\end{abstract}

Palabras clave: Disciplinariedad, transdisciplinariedad, complejidad, Pedagogía, educación.

\begin{abstract}
The crisis of hegemonic models of knowledge and the request from the complex educational reality impugn the work of sciences of education since they are limited by the simplicity paradigm in its disciplinary expression. This article discusses about the modes how has been understood the comprebension of education and it provides some elements to think a pedagogy from the complexity, in other words, a pedagogy as a transdisciplinary construction.
\end{abstract}

Keywords: Discipline, Transdisciplinary, Complexity, Pedagogy, Education.

Recibido el 27 de mayo de 2013 y aprobado el 20 de junio de 2013

1 Estudiante Licenciatura en Educación Básica con énfasis en Ciencias Sociales. Universidad Pedagógica Nacional (UPN), Bogotá. Correo electrónico: kevin_danielrozo065@hotmail.com

2 El presente es un artículo de reflexión resultado de la investigación desarrollada en el Seminario Historia y Epistemología de la Pedagogía de la Licenciatura en Educación básica con Énfasis en Ciencias Sociales de la Universidad Pedagógica Nacional. Bogotá, Colombia. 


\section{"Cada método es un lenguaje y la realidad responde en la lengua}

en que es preguntada"

Boaventura de Sousa Santos

\section{"Solo una inteligencia que dé cuenta de la dimensión planetaria de los conflictos actuales podrá hacer frente a la complejidad de nuestro mundo"}

Carta a la transdisciplinariedad

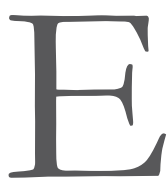

n los albores del siglo XX se empieza a generar un desencanto generalizado por la ciencia moderna, ello llevó a algunos grupos representativos de la intelectualidad a repensar la realidad y sus formas de ser abordada, pues ésta se había vuelto compleja y, así mismo, la forma de estudiarla debería ser modificada. En este panorama se pone en cuestión el culto a la disciplinariedad — propio de la ciencia moderna- y así emergen nuevas maneras de estudiar la realidad, a saber, la interdisciplinariedad, y más recientemente la transdisciplinariedad. Dichas perspectivas constituyen nuevos niveles de realidad que responden a las demandas de la sociedad contemporánea. En medio de este debate epistemológico emerge el Grupo de Historia de la Práctica Pedagógica en Colombia, que dentro de sus objetivos investigativos tiene la pretensión de otorgarle un estatuto epistemológico de disciplina a la Pedagogía en tanto que ciencia social. En el presente escrito pretendo hacer una revisión crítica de esa apuesta y en un segundo momento pondré de presente la posibilidad de otorgarle un estatuto epistemológico a la Pedagogía como construcción transdisciplinar.

\section{Un regreso a los debates} de la ciencia moderna

El siglo XIX constituyó el icono del progreso de la ciencia y técnica moderna. Los procesos de industrialización masiva y la mundialización del sistema capitalista-imperialista fueron la condición de posibilidad para la consolidación del paradigma positivista, cuyo imperativo es la medida, la exactitud, la objetividad, la verificabilidad, la ley y la negación arbitraria de cualquier otro tipo de conocimiento, siendo este último tachado de irracional e ilegítimo. En el momento de mayor madurez de las ciencias naturales emergen las ciencias sociales como subproducto de las primeras, enmarcadas en la figura del monismo metodológico que pretendía aplicar los mismos esquemas analíticos formales para estudiar los fenómenos naturales y los fenómenos sociales, bajo la pretensión de axiomatizar —al igual que la naturaleza- la sociedad.

Empero, en las ciencias sociales con el paso del tiempo empezó a circular una desconfianza por el paradigma dominante - positivista - y de esta manera surgió una nueva apuesta que apelaba a la interpretación. El reconocimiento del observador en la investigación y la importancia de la comprensión de los hechos sociales constituían un proceso de "atraso" frente a la exactitud de las ciencias naturales, no obstante, ello representaría una crisis del modelo hegemónico de conocimiento (de Sousa Santos, 2009).

Estas dos posturas dan cabida a la dualidad de la ciencia moderna, que separa en una dicotomía irreconciliable las relaciones sujeto-objeto, naturaleza-cultura, mentemateria, observador-observado, estructuraagencia, colectivo-individual, etc. La apuesta desde la perspectiva analítica de la transdisciplinariedad presupone superar dichas ambigüedades. Esto lo trataré más delante.

Sin embargo, en medio de tales tensiones epistemológicas seguía intacto el culto a la disciplina, entiéndase un cuerpo teórico,

Polisemia No. 15, 43 - 51. Más allá de la disciplinariedad. Elementos para pensar la Pedagogía como construcción [...]. Bogotá, ISSN: 1900-4648. Enero - junio de 2013 
cualitativamente diferente de otros, en tanto que se ocupa de un fenómeno distinto, específico, que presenta leyes propias en su desarrollo (Setién Quesada, 1996). La ciencia moderna, en medio de sus contradicciones, mantenía la pretensión de vigilar las fronteras de sus disciplinas, tanto en objeto como en métodos de estudio. El progreso de la ciencia y técnica modernas dependía de la fragmentación y especialización de los campos disciplinares.

Por otra parte el debate continuaba. A comienzos siglo $\mathrm{XX}$, los constructos teóricos de las ciencias naturales, en particular de la Física, se aproximaron a ese desencanto por el paradigma dominante que ya había afectado a las ciencias sociales y que, pareciera ser, deslegitimaba todos los progresos logrados hasta la época. Por un lado, la teoría de la relatividad de Einstein puso en duda la universalidad de las leyes, relativizando las relaciones espacio-tiempo-materia. Y no solo eso, además relativizó los procesos de verificabilidad frente a lo definible. Por otro lado, la física cuántica transformó la relación objetiva de la Modernidad entre sujeto y objeto, reivindicando el papel de la subjetividad y del sujeto participante en el proceso investigativo.

Así pues, es evidente la crisis del criterio de verdad que respaldaba y auto legitimaba la ciencia moderna. ¡Ni universalidad de las leyes, ni verificabilidad, ni objetividad! Ahora la ciencia positiva volteaba su mirada a las ciencias sociales.

\section{Interdisciplinariedad, complejidad y transdisciplinariedad}

La posguerra trajo consigo una reestructuración de la política mundial. La bipolarización del mundo y sus heces - el tercer mundo- (Gallardo, 1991), contemplaron en tan solo 25 años el mayor crecimiento demográfico que haya visto la historia, un tremen- do desarrollo de las fuerzas productivas y "las maravillas" del crecimiento económico. La hegemonía de los Estados Unidos se hacía presente en el plano material y simbólico, y para asegurar su perpetuación se empezaron a desarrollar los llamados estudios de área. Estos consistían en una serie de investigaciones en territorios estratégicos de Nuestra América, Asia menor, norte de África, etc., que desde la perspectiva analítica de diferentes disciplinas sociales, a saber, Geografía, Sociología, Lingüística etc., tenían como fin proporcionar una perspectiva holística de las realidades investigadas en aras de controlar — material y simbólicamente- determinadas regiones del mundo. Así, la expansión económica reforzó la legitimación global de las ciencias sociales (Wallerstein, 1996).

Es precisamente esta intencionalidad de visión totalizadora de la realidad la que obliga a las diferentes disciplinas a entrar en diálogo, esto es, a encontrar puntos de convergencia entre las disciplinas en la que cada una aporta sus problemas, conceptos y métodos de investigación (Pérez \& Setién, 2008), dicho de otra manera: la interdisciplinariedad. Ésta se afianza con la constitución de la Unesco como "organización para la cooperación internacional", pues la masiva financiación a los emergentes estudios culturales permitió su desarrollo y madurez. De esta manera, se consolidan la etnografía, los estudios orientales y demás construcciones representativas de la interdisciplinariedad. El estudio sistemático de las categorías etnia, género, raz̧a, religión, diversidad de subjetividades, etc., constituye el horizonte teórico que guía tales estudios, los que sólo es posible comprender desde una perspectiva totalizadora. En suma, la vieja creencia de que el campo disciplinar definía los problemas de estudio queda devaluada en la perspectiva analítica interdisciplinar. Contrariamente, ésta supone que son los problemas específicos de la realidad los que definen las perspectivas disciplinares desde donde han de ser estudiados, en consonancia, el diálogo entre campos disciplinares se hace inevitable. 
Situados de forma kairológica - no cronológica- en los albores del presente siglo, la nueva organización del sistema mundo (Wallerstein, 1996) y todas las relaciones objetivas y subjetivas que de allí derivan, es evidente que atravesamos un tiempo de crisis. Esta crisis hoy rebasa los límites de una simple oscilación del modo de producción capitalista o de una serie de decisiones políticas; estamos presenciando un tiempo de crisis sistémica, una crisis cultural, axiológica, material y, por supuesto, científica. La realidad se ha complejizado, y ello demanda una revisión y reestructuración del modo de estudiarla, esto es, una comprensión compleja de la realidad, pues: "solo una inteligencia que dé cuenta de la dimensión planetaria de los conflictos actuales podrá hacer frente a la complejidad de nuestro mundo" (Carta a la transdisciplinariedad, 1994). La complejidad, en este sentido, no es más que la imposibilidad de comprender la realidad en su totalidad, de descubrir y sistematizar eso ordenado, de ordenar eso que se presenta como caos. La realidad no es ni cosmos ni caos, es ante todo una unidad compleja (Morin, 2011). Así pues, la complejidad es una palabra problema y no una palabra solución (Idem.). Ahora bien, eso no quiere decir que no podamos construir modelos teóricos para aproximarnos a la comprensión de eso real. Así es la transdisciplinariedad.

Entonces, la interdisciplinariedad, la complejidad y la demanda de abordar nuevos niveles de realidad son condiciones de posibilidad para la emergencia de la transdisciplinariedad. El término fue utilizado por primera vez por Piaget en el congreso de la OCDE de 1970, cuando propone adoptar la transdisciplina en cuanto que perspectiva externa a la lógica disciplinaria. Pero sería hasta la década de los noventa cuando una serie de teóricos a nivel global considerarían sumarse a esta corriente intelectual y ello se vería cristalizado en la Carta a la transdisciplinariedad. Esta pone de presente los principios fundamentales de la actitud transdisciplinaria, así como sus apuestas teóricas y su posicionamiento político.

La transdisciplina es una construcción epistemológica independiente de la lógica disciplinar (Zavala, 2010), que tiene como intención:

Superar la fragmentación de los conocimientos, más allá del enriquecimiento de las disciplinas con diferentes saberes -multidisciplinar-y el intercambio epistemológico y de métodos científicos de los saberes -interdisciplinar-. Lo que caracteriza a la transdisciplinariedad no es la realidad interactuante sino totalizadora (Pérez \& Setién, 2008).

Es necesario citar el cuarto artículo de la Carta a la transdisciplinariedad: "La clave de la bóveda de la transdisciplinariedad reside en la unificación semántica y operativa de las acepciones a través de una nueva mirada sobre la relatividad de las nociones de definición y objetividad" (1994). Dicho de otra manera, la condición de transición entre interdisciplinariedad y transdisciplinariedad consiste en la hibridación de las fronteras disciplinares de la primera, cristalizadas en la institución de un nuevo entramado semántico de conceptos, métodos y esquemas analíticos formales. En este orden de ideas, la transdisciplina se genera al descubrir nuevos objetos de estudio que no pueden ser estudiados desde una perspectiva disciplinaria (Zavala, 2010). De allí que la transdisciplinariedad sea un concepto que emerge de un contexto de aplicación concreto, con sus propias estructuras teóricas, métodos de investigación y modos de práctica (Gibbons, 1994).

En al campo ideológico, es decir, en lo que respecta a las estrategias de representación y legitimación vale la pena traer a colación algunas de las diferencias que identifica Zavala entre el paradigma disciplinario (A) y el transdisciplinario (B), donde A hará referencia al primero y $\mathrm{B}$ al segundo - por cuestiones explicativas-. Mientras A representa y legitima la ciencia, B prefiere hablar de teorías texto-intertexto; A se refiere a "tener razón", cuando B apuesta por "ser razonable"; A, racionalidad instrumental discriminativa, B, Inteligibilidad: "razón inferencial"; mientras A sigue hablando de objetividad, B propone un regreso a la subjetividad y a

Polisemia No. 15, 43 - 51. Más allá de la disciplinariedad. Elementos para pensar la Pedagogía como construcción [...]. Bogotá, ISSN: 1900-4648. Enero - junio de 2013 
la intersubjetividad; A, verdades absolutas, B, "verdades contextuales: conjeturas"; cuando A sigue separando sujeto y objeto, B reivindica el observador implicado; en tanto A defiende la dialéctica, B apologiza la dialógica; A comprende el lenguaje científico como un espejo de la realidad, para $\mathrm{B}$ el lenguaje es interpretación de la realidad; A cree en la validez universal, mientras $\mathrm{B}$ considera que todo universalismo es contingente; cuando A apela al uso normativo de nombres nuevos para conceptos nuevos, B prefiere la jurisprudencia lexical: reciclaje nominal y nombres nuevos para lo nuevo, de allí la transformación semántica en el proceso de hibridación disciplinar (Zavala, 2010).

En un sentido más amplio,

rigor, apertura y tolerancia son las características fundamentales de la actitud y visión transdisciplinaria. El rigor en la argumentación, que toma cuenta de todas las cuestiones, es la mejor protección respecto a las desviaciones posibles. La apertura incluye la aceptación de lo desconocido, de lo inesperado y de lo imprevisible. La tolerancia es el reconocimiento del derecho a las ideas y verdades contrarias a las nuestras (Carta a la transdisciplinariedad, 1994).

\section{Hacia una Pedagogía como construcción transdisciplinar}

Las ciencias de la educación aparecen a principios del siglo XX, cuando se tuvo la pretensión de convertir la educación en una ciencia. Las ciencias de la educación son un conjunto de disciplinas que tienen en común el estudio de las situaciones y de los hechos educativos tanto a nivel micro como macro educativo (Zuluaga et al., 2003, p. 21).

Así, aportes teóricos provenientes de la Sociología de la educación, la Antropología de la educación, la Psicología de la educación, la Administración educativa - y según Zuluaga et al. (1998), la Pedagogía y la Didáctica- empezaron a construir un nuevo discurso que contenía en sí mismo una gran carga interdisciplinar.
El Grupo de Historia de la Práctica Pedagógica en Colombia inicia su trabajo de epistemologización de la Pedagogía haciendo una crítica a la perspectiva anteriormente expuesta - ciencias de la educación- pues la apuesta agenciada por el grupo consiste en independizar la Pedagogía y además concederle a ésta el papel más importante entre las ciencias de la educación, atribuyéndole — para su legitimación-el estatuto de disciplina.

El conjunto de disciplinas que conforman las ciencias de la educación, con excepción de la pedagogía y de la didáctica, se ocupan de la educación a su manera, es decir, mediante nociones, conceptos y métodos propios de una disciplina macro, por ejemplo la sociología de la educación, hace parte de la sociología, la psicología de la educación corresponde al campo de la psicología (Zuluaga et al., 2003, pp. 22-23).

Esto es evidente pues ni la Pedagogía ni la Didáctica poseen un conjunto sistematizado de teorías, conceptos y esquemas analíticos propios que le permitan hacerlo por separado, como bien lo podría hacer la Sociología, la Psicología y la Filosofía educativa. Pues la Pedagogía configura y enriquece su discurso desde la apropiación e hibridación de los saberes provenientes de otras disciplinas sociales que se piensan el problema de la educación.

Ahora bien, esta tensión planteada por el Grupo de Historia de la Práctica Pedagógica en Colombia supone que en ese estado de cosas surge un proceso de enrarecimiento de la Pedagogía, que trae como consecuencias la conceptualización desarticulada de los saberes sobre el problema educativo, la atomización, la subordinación de la Pedagogía y su instrumentalización, lo que termina reduciéndola a un simple hecho operativo. En Zuluaga et al. (2003), la Pedagogía se constituye como disciplina sobre las bases de la práctica pedagógica y el saber pedagógico. La primera hace referencia a las experiencias propiamente dichas del maestro en su campo de acción: la enseñanza. La segunda constituye un saber enseñar, es decir, las formas de transmisión institucional del saber social, dicho saber 
es elaborado y operacionalizado por los maestros como agentes participantes en el proceso pedagógico y que tiene como objeto central la reflexión sobre la práctica pedagógica y se concreta operativamente en los métodos de enseñanza (Zuluaga et al., 2003). Tal situación se torna problemática en medio de la empresa de epistemologización de la Pedagogía como disciplina, por cuanto: "el campo del saber pedagógico está disgregado y sus criterios de verdad son puestos en crisis por los desplazamientos, y la multiplicación de sus objetos en las nuevas disciplinas" (Zuluaga et al., 2003, p. 23). De esta manera, la Psicología de la educación se ha apropiado del aprendizaje — en tanto objeto de estudio-, la Sociología de la educación hace lo propio con la institución educativa - la escuela-, la Administración educativa realiza una lectura de la escuela en términos de empresa, etc. y la Pedagogía —al igual que la Didácticaquedan sin ningún trozo de la torta educativa. De allí la necesidad de entablar el diálogo entre diferentes disciplinas sociales para ir construyendo y legitimando su propio discurso.

En materia epistemológica, Zuluaga et al. son persistentes en la necesidad de otorgarle un estatuto disciplinar a la Pedagogía:

Todos estos trabajos requieren el reconocimiento de la pedagogía como disciplina, lo que no significa establecer límites a la manera de un cerco, debemos considerar que los conceptos no conocen frontera epistemológicas, ellos se relacionan de una teoría a otra adquiriendo en cada una un significado peculiar, y un modo diferente de inserción en el conjunto de conceptos que conforman una disciplina. A la luz de este principio asumimos la relación de la pedagogía con otras disciplinas, convencidos desde luego que las disciplinas avanzan a partir de conceptos y métodos que pueden provenir de otros campos de conocimiento (1998, p. 31).

Es evidente que en esta apuesta ya se empieza a flexibilizar el cuidado de la frontera, por cuanto se empieza a asumir la Pedagogía como una construcción que proviene del diálogo entre diferentes disciplinas sociales que se piensan el problema educativo. De esta manera, se presenta como menester pasar de la flexibilización entre fronteras disciplinares a la hibridación de los conceptos, las teorías, los métodos $\mathrm{y}$ todos aquellos aspectos que agenciados por el emergente discurso pedagógico podrían ser condición de posibilidad para la aproximación — cuando menos- de la comprensión de la realidad educativa, que es ante todo una unidad compleja, ello implica a su vez un abordaje complejo, y éste solo es posible a partir de una Pedagogía como construcción transdisciplinar.

Siendo un poco más meticuloso, Zuluaga et al. (2003) se basan en Herbart para argumentar las posibilidades de un estatuto disciplinar para la Pedagogía.

En una reflexión sobre el carácter científico de la pedagogía hay que tener en cuenta lo que advertía Herbart en el sentido de que ésta vuelva con mayor precisión posible a sus conceptos autónomos y "cultive más un pensar autóctono; de modo que se constituya en centro de un círculo de investigación" (Herbart citado por Zuluaga et al., 2003, p. 28).

Esto resulta claramente imposible, por cuanto la Pedagogía, al depender del diálogo entre disciplinas, no puede "cultivar un pensamiento autónomo" en el sentido disciplinar, pues éste presupone la exclusividad de los saberes. Dicho de otra manera, la Pedagogía es producto del diálogo histórico que se ha dado entre las ciencias sociales sobre la educación y que se empieza a cristalizar en un discurso propio que desde diferentes perspectivas analíticas — sociológica, psicológica, filosófica, antropológica, etc.han intentado comprender la realidad educativa de forma totalizadora; sin embargo, ello tiene lugar sólo al concebir una Pedagogía pensada desde la complejidad.

En este punto de la discusión emerge una paradoja, pues: "en comparación con otros discursos científicos la pedagogía se ha formado relativamente tarde, apoyándose en teorías específicas más o menos ya constituidas de otras ciencias sociales, como la psicología o la sociología —o aún la economía_-" (Bedoya

Polisemia No. 15, 43 - 51. Más allá de la disciplinariedad. Elementos para pensar la Pedagogía como construcción [...]. Bogotá, ISSN: 1900-4648. Enero - junio de 2013 
Madrid citado por Zuluaga et al., 2003, p. 83). Mientras se piensa en clave del paradigma moderno de la ciencia, es evidente el malestar de la Pedagogía frente a las otras disciplinas sociales por su inmaduro estado positivo; empero, abordando el problema desde un nuevo nivel de realidad, la Pedagogía desde su emergencia ya había superado el estado positivo de la ciencia y se aproximaba más a una lectura compleja de la realidad, esto es, como construcción transdisciplinar.

Más recientemente, Alejandro Álvarez Gallego, miembro del Grupo de Historia de la Práctica Pedagógica en Colombia, en un texto inédito (2010) vuelve a la reflexión epistemológica de la pedagogía y manifiesta un desencanto por la pretensión disciplinar que el grupo le había pretendido dar en el pasado. Para Álvarez, "los espacios culturales serían tan o más pedagógicos que la escuela" (2010). En este sentido, la Pedagogía ha transgredido las fronteras de la escuela y se ha abierto a todos los ámbitos de la cultura, entonces, "un concepto sobre la pedagogía debería incluir todos los lugares públicos que hoy moldean, forman, educan y socializan a las personas incluyendo los centros comerciales" (Idem.). En suma, para Álvarez; la Pedagogía sería una suerte de discurso secundario que cumple la función de reproducir y recontextualizar discursos primarios. La Pedagogía, al no tener un campo teórico propio, entra en un desencanto por la disciplinariedad - tan costosa para la ciencia moderna- y de esta manera, se constituye en un dispositivo que selecciona, transforma, reorganiza y reubica los saberes disciplinares que actúan en todas las esferas de la cultura. En ese orden de ideas, la Pedagogía agenciaría un control discursivo de carácter legitimador, que se traduciría en lo que se enseña - y cómo- y lo que no se enseña; en lo que se reproduce y lo que no se reproduce (Idem.).

Ahora bien, la Pedagogía, en el sentido en que la plantea Álvarez, quedaría reducida a un papel subsidiario de los discursos primarios
— cuerpos especializados de conocimiento-y no tendría, por ende nada que decir acerca de la realidad educativa. Sin embargo, su argumento puede ser recontextualizado en el campo de la Didáctica.

Conciliando, yo propondría la didáctica como un puente articulador entre la teoría y práctica concretas de la reproducción y recontextualización de discursos primarios —estos bien pueden ser disciplinas como la Biología, la Física, etc.- y que de manera particular demanda una reflexión especializada desde las didácticas específicas, que siendo sus supuestos y maneras de proceder de diferente naturaleza, siguen orientadas a los mismos objetivos macro ya planteados en el ser de la Didáctica.

En suma, el campo reflexivo y totalizador sobre la realidad educativa —en todos los ámbitos de la cultura - queda abierto para la Pedagogía como construcción transdisciplinar. Ello se debe a que la fragmentación por especialización del estudio de tal realidad, que es compleja, termina imposibilitando una reflexión holística que logre comprender la génesis, estructura, funcionamiento, contradicciones, contingencias, etc., que configuran la realidad educativa. Ello supone, desde el principio de la tolerancia, valorar los aportes que la Sociología, la Psicología, la Historia y de manera general las ciencias sociales han hecho a la reflexión sobre la educación, y generar un diálogo relacional entre éstas como condicio sine qua non para la bibridación conceptual y metodológica que deriva, en últimas, en la configuración de una Pedagogía pensada desde la complejidad. Es decir, un discurso reflexivo capaz de afrontar los retos que impone la realidad educativa que se ha hecho imposible de comprender desde la fragmentación, que demanda una lectura totalizadora, a saber, una Pedagogía como construcción transdisciplinar.

Todo esto no quiere decir que las ciencias de la educación deban desaparecer. No. Por el contrario, éstas siguen teniendo importancia para la racionalización de la realidad educativa, pero de otra manera. Las ciencias de la educación 
pierden su posición hegemónica en la empresa de comprensión de la realidad y se convierten, de otra parte, en reflexiones alimentadoras que nutren desde el ser parcelado de la realidad educativa a la Pedagogía, que en tanto transdisciplina rectora de la reflexión educativa tiene como función la apropiación, relación, diálogo, bibridación y resignificación de dichos nutrientes que, en último término, se erige como posibilidad de aproximación a la comprensión de dicha unidad compleja: la educación.

Pensar la Pedagogía desde la complejidad y, en particular, como construcción transdisciplinar no es una receta que yo quiera imponer, o que se pueda llevar a cabo sólo agregando los ingredientes indicados; es, más bien, una alerta, un llamado a la comunidad científica y educativa a que piense de otra manera el modo en que hemos abordado la realidad educativa, es reconocer que hoy tenemos más que nunca múltiples miradas parceladas, pero ninguna reflexión totalizadora. Necesitamos construir nuevos modelos, no sólo para comprender dicha unidad compleja, sino también para operar en ella, transformarla, de eso se trata. Necesitamos quitarnos las gafas de la simplicidad, se trata de afrontar los retos que nos pone de presente la realidad educativa, se trata de complejizar su estudio. No podemos seguir abordando lo complejo por lo simple; se trata de abordar lo complejo por lo complejo.

Para concluir, quisiera citar el segundo artículo de la Carta a la transdisciplinariedad:

El reconocimiento de la existencia de diferentes niveles de realidad, regidas por diferentes lógicas, es inherente a la actitud transdisciplinaria. Toda tentativa de reducir la realidad a un solo nivel, regido por una única lógica, no se sitúa en el campo de la transdisciplinariedad (1994).

No podría sintetizarse mejor, la apuesta y el compromiso que nos queda ahora es luchar contra todo intento de reducción de la realidad educativa, hemos de diseñar estrategias de integración en equipo ${ }^{3}$ — sólo por dar un ejemplo- (Klein, 1996, citado por Zavala, 2010) para complejizar el estudio de dicha realidad, ello implica por supuesto empezar a comprender la Pedagogía como una construcción transdisciplinar.

3 Algunas de ellas, según Klein, podrían ser:

1. Definir el objetivo común.

2. Determinar el conocimiento necesario, incluyendo modelos, tradiciones y bibliografía.

3. Desarrollar un marco de integración y preguntas estratégicas.

4. Especificar lo que es necesario estudiar.

5. Establecer negociación de roles en el trabajo de equipo.

6. Reunir información disponible e investigar nueva información.

7. Resolver conflictos disciplinarios creando un vocabulario común.

8. Mantener comunicación a través de técnicas de integración.

9. Sintetizar toda la información, evaluar su relevancia y determinar un patrón general.

10. Decidir la administración futura del proyecto.

Polisemia No. 15, 43 - 51. Más allá de la disciplinariedad. Elementos para pensar la Pedagogía como construcción [...]. Bogotá, ISSN: 1900-4648. Enero - junio de 2013 


\section{Referencias bibliográficas}

Álvarez, A. (2010). Educación Popular, Pedagogías Críticas y Estudios Culturales: Confuencias teóricas y trayectos políticos, Colombia. 1970-1990. (Mimeo).

Carta a la transdisciplinariendad. (Noviembre, 1994). Convento de Arrábida. Recuperado de: http://www.filosofia.org/cod/ c1994tra.htm.

De Sousa Santos, B. (2009). Una epistemología del Sur. México D.F.: Siglo XXI.

Gallardo, H. (1991). Crisis del socialismo histórico, ideologías y desafíos. San José: Del.

Gibbons, M. et al. (1997). La nueva producción del conocimiento. Barcelona: Pomares.
Morin, E. (2011). Introducción al pensamiento complejo. Barcelona: Gedisa.

Pérez, N. \& Setién, E. (2008). La interdisciplinariedad y la transdisciplinariedad en las ciencias: una mirada a la teoría bibliológico-informativa. Recuperado de: http://bvs.sld.cu/revistas/ aci/vol18_4_08/aci31008.htm.

Wallerstein, I. (1996). Abrir las ciencias sociales. Informe de la Comisión Gubelkian D.F.: Siglo XXI.

Zavala, L. (2010). Transdisciplinariedad. Principios generales. México D.F.: UAM-X.

Zuluaga, O. Echeverri, A., Martínez, A., Restrepo, S., \& Quinceno, H. (marzo, 2003). Educación y Pedagogía una diferencia necesaria. Revista Educación y Pedagogía, 13, 4-9. 